\title{
Transparency and Openness Promotion Guidelines for $\mathrm{HCl}$
}

Lewis L. Chuang

Department for Perception,

Cognition, and Action,

Max Planck Institute for

Biological Cybernetics

Max Planck Ring 8, 72076 ,

Tübingen, Germany

lewis.chuang@tuebingen.mpg.de

\section{Ulrike Pfeil}

HCI Group,

Department for Computer \&

Information Sciences,

University of Konstanz

D 73, 78457 Konstanz

ulrike.pfeil@uni-konstanz.de

\begin{abstract}
This special interest group addresses the status quo of $\mathrm{HCl}$ research with regards to research practices of transparency and openness. Specifically, it discusses whether current practices are in line with the standards applied to other fields (e.g., psychology, economics, medicine). It seeks to identify current practices that are more progressive and worth communicating to other disciplines, while evaluating whether practices in other disciplines are likely to apply to $\mathrm{HCl}$ research constructively. Potential outcomes include:

(1) a review of current $\mathrm{HCl}$ research policies, (2) a report on recommended practices, and (3) a replication project of key findings in $\mathrm{HCl}$ research.
\end{abstract}

\section{Author Keywords}

open science; replication; methods.

\section{Background and Motivation}

In 2015, a landmark paper was published that summarized the efforts of 270 researchers in replicating 100 studies, published across 3 highly-esteemed psychology journals

[2]. It found that approximately a third of previously reported effects replicated in subsequent studies. Thus, it concluded that the critical features of scientific enterprise, namely transparency, openness, and reproducibility, were significantly undermined in actual research practice. 


SIDEBAR 1:
TOP GUIDELINES [9]
Citation standards
Replications
Transparency: Data
Transparency: Analytic meth-
ods
Transparency: Research
materials
Transparency: Design \&
analysis
Preregistration: Studies
Preregistration: Analysis

Since then, digital instruments have been developed to faciliate the documentation and dissemination of the progressive stages of research projects, with the express goal of facilitating open research. In particular, the Open Science Framework (osf.io) provides a digital platform that allows researchers to document and time-stamp progress in their research projects as well as share relevant data, code, and analytical tools. Cogent arguments have been made in favor of the open dissemination of such auxiliary research materials (e.g., [7, 9]). Doing so ensures the replication of foundational findings, whenever necessary, that the success of new research proposals heavily rely on. Moreover, it promotes interdisciplinary research between, otherwise, disjointed communities that may not communicate published findings but may, nonetheless, be interested in shared data and methods. There is little doubt that an emphasis on transparency, openness, and reproducibility will enhance public (as well as collegial) trust in the scientific process [9]. To this extent, funding agencies are increasingly supportive of the adoption of open science practices in the projects they sponsor (e.g., [10]). In line with this, advocates of recognized that the adoption of Open Science practices depend on, not only on the ready availability of tools but also, a reasonable recognition of the status quo and the effort to improve this, and the appropriate incentivisation of this effort.

This has given rise to the formalization and advocacy of "Transparency and Openness Promotion" (TOP) guidelines that strive to advance research [9]. Eight standards and three levels of increasing stringency comprise TOP guidelines (see Sidebar 1 and next section for details), with the primary objective of incentivising transparency in research practices. To date, more than 5000 journals and institutions have signed up to review their practices in accordance to TOP guidelines; the Association of Computing Machin- ery and, notably, its Special Interest Group for ComputerHuman Interaction (SIGCHI) are non-signatories.

Arguably, the human-computer interaction $(\mathrm{HCl})$ community has pioneered many of the open practices advocated thus far. In fact, many $\mathrm{HCl}$ researchers actively share their code for development on various platforms (e.g. GitHub) to increase early adoption, as well as to strengthen the impact and visibility of their work. $\mathrm{HCl}$ Journals and conferences actively encourage researchers to submit supplementary material, such as "survey text, experimental protocols, source code, and data, all of which can help with replicability of [the] work." [1]. Further, $\mathrm{HCl}$ articles are shared on open access journals (doaj.org) or submitted to ArXiv (arxiv.org) that increase the visibility of work beyond the domain of $\mathrm{HCl}$ research. Recent years have also witnessed significant efforts in promoting the value of replication studies [11] [4]. Open practices and their practical value to $\mathrm{HCl}$ research have also been strongly encouraged [4]. Besides this, there is a growing appreciation of statistical reporting practices that promote transparency and openness, as evidenced by the popularity of workshops and SIGs on this theme $[5,6]$.

This raises the question: Are formal guidelines for transparency and openness unnecessary or, worse, counterproductive to the advancement of $\mathrm{HCl}$ research?

\section{TOP guidelines}

The Center of Open Science advocates eight standards (i.e., TOP guidelines; see Sidebar 1) that supports the translation of Open Science values (i.e., transparency, openness, and reproducibility) into concrete and tangible actions. Signatories (e.g., journals, professional societies, funding agencies, etc.) to TOP guidelines commit themselves to reviewing existing policies and procedures, and 


\section{SIDEBAR 2: SIG AGENDA}

\section{0 mins}

Welcome and Introduction (5 mins)

\section{5 mins}

Invited talk by COS representative (15 mins)

\section{0 mins}

Debate: $\mathrm{HCl}$ does not require

TOP guidelines

(20 mins)

\section{0 mins}

Breakout discussion on TOP

guidelines (20 mins)

\section{0 mins}

Present decision on current $\mathrm{HCl}$ standards with regards to TOP guidelines and recommended next steps (15 mins

75 mins

Summary statement (5 mins)

80 mins

END OF SIG determine the current level of stringency (i.e., levels 0-3) of current practices. TOP guidelines serve to incentivise the research community to formally adopt practices that promote openness and transparency. They can be summarized in three groups:

Reward for open practice: Besides published communications, citation standards could include citations of data, code and research material, to credit them as original intellectual contributions. Stable versions of repositories should be cited, which provides a formal acknowledgement of their intellectual contributions to the community. Also, publication venues could actively encourage the submission of replication studies, especially those with fundamental implications for high impact research domains. These practices will allow for self-correction as well as identify promising research directions [9].

Transparency: TOP guidelines identify at least four types of contributions that deserve recognition in their own right. These should be openly shared independent of the research publication itself. They are: Data, Analytic methods, Research materials, Design and analysis. Such contributions allow others to understand the detailed procedures in analyzing the data collected in order to generate the reported results. This includes providing access to research prototypes under a license that permits use, modifications, improvements, or derivations from existing source code [8].

Furthermore, the research material standard promotes to provide all elements of that methodology and data sharing standards that make data available in common trusted repositories (e.g. the Open Science Framework). Finally, the analytic methods standard promote the publication and citation of the code and the statistical models and simulations used in the research [9]. Access to raw datasets without restrictions allow reuse and aggregation of research data from multiple resources for extended analysis [8]. This will reduce uninformative variance across different studies and ensure that best practices are repeated. Data sharing will also promote new discoveries as well as contribute to modeling and simulation efforts.

Pre-registration: Pre-registrations of studies and analyses are strongly encouraged to distinguish between research that are confirmatory (hypothesis-testing) and exploratory (hypothesis-generating). Researchers are encouraged to submit their research proposals and associated hypotheses for review prior to data-collection. Research proposals that pass peer-review are accepted prior to data collection, if the protocols are observed. This procedure is expected to minimize the abuse of inferential statistics [3]

\section{Structure of the SIG and Outcome}

The objective determine whether the TOP guidelines apply to advance current $\mathrm{HCl}$ research practices. The opinions raised before, during, and after this SIG is documented as an Open Science Framework project (http://osf.tophci.org/). This document will inform the COS as well as the executive committee of ACM SIGCHI.

Sidebar 2 provides a brief structure of the SIG meeting. It will commence with an invited talk by a representative from the Center for Open Science (COS). This talk will introduce the COS, tools for Open Science, the challenges in implementing recommended practices, and the outcome of incentivising Open Science. Next, there will be an Oxfordstyle debate on the proposition: "Transparency and Openness Promotion guidelines are unnecessary and potentially counterproductive to the advancement of $\mathrm{HCl}$ research.". Two three-member panels will separately represent an affirmative and negative position on the proposition, independent of their own beliefs. This is based on the opinions 
shared prior to the meeting via the OSF project. Following this, 4 independent groups will be formed to address two TOP guidelines each. The goal for each group will be to determine the current level of each standard and to discuss whether it applies to $\mathrm{HCl}$ research. Discussants will address the relevance of TOP guidelines and how they might be applied or modified accordingly. Each group will subsequently present the results of their discussion. The SIG meeting will conclude with an invitation for continued contribution to an open collaborative document on the applicability of TOP guidelines to ACM SIGCHI interests.

The discussion could yield positive $\mathrm{HCl}$ practices that could be recommended to COS. Also, an interest in TOP practices could inspire a new research domain, namely developing better human-computer interfaces for facilitating the adoption of Open Science practices by scientists.

\section{REFERENCES}

1. CHI 2018, Montreal, Canada: Papers (Preparing and Submitting Your Paper).

https://chi2018.acm.org/authors/papers/. (????) Accessed: 2018-01-15

2. Open Science Collaboration and others. 2015. Estimating the reproducibility of psychological science. Science 349, 6251 (2015), aac4716.

3. Megan L Head, Luke Holman, Rob Lanfear, Andrew T Kahn, and Michael D Jennions. 2015. The extent and consequences of $\mathrm{p}$-hacking in science. PLoS biology 13, 3 (2015), e1002106.

4. Kasper Hornbæk, Søren S Sander, Javier Andrés Bargas-Avila, and Jakob Grue Simonsen. 2014. Is once enough?: on the extent and content of replications in human-computer interaction. In Proceedings of the 32nd annual ACM conference on Human factors in computing systems. ACM, 3523-3532.
5. Matthew Kay, Steve Haroz, Shion Guha, and Pierre Dragicevic. 2016. Special Interest Group on Transparent Statistics in $\mathrm{HCl}$. In Proceedings of the $2016 \mathrm{CHI}$ Conference Extended Abstracts on Human Factors in Computing Systems (CHI EA '16). ACM, New York, NY, USA, 1081-1084. DOI :

http://dx.doi.org/10.1145/2851581.2886442

6. Matthew Kay, Steve Haroz, Shion Guha, Pierre Dragicevic, and Chat Wacharamanotham. 2017. Moving Transparent Statistics Forward at $\mathrm{CHI}$. In Proceedings of the $2017 \mathrm{CHI}$ Conference Extended Abstracts on Human Factors in Computing Systems (CHI EA '17). ACM, New York, NY, USA, 534-541.

DOI : http://dx.doi.org/10.1145/3027063.3027084

7. Peter Kraker, Derick Leony, Wolfgang Reinhardt, and Günter Beham. 2011. The case for an open science in technology enhanced learning. International Journal of Technology Enhanced Learning 3, 6 (2011), 643-654.

8. Peter Murray-Rust. 2008. Open data in science. Serials Review 34, 1 (2008), 52-64.

9. Brian A Nosek, George Alter, George C Banks, D Borsboom, Sara D Bowman, Steven J Breckler, Stuart Buck, Christopher D Chambers, G Chin, G Christensen, and others. 2015. Promoting an open research culture. Science 348, 6242 (2015), 1422-1425.

10. OpenUp. 2018. OpenUp Project. (2018). http://openup-h2020.eu/

11. Max L Wilson, Wendy Mackay, Ed Chi, Michae Bernstein, Dan Russell, and Harold Thimbleby. 2011. RepliCHI-CHI should be replicating and validating results more: discuss. In CHI'11 Extended Abstracts on Human Factors in Computing Systems. ACM, 463-466. 